\title{
The beryllium atom and beryllium positive ion in strong magnetic fields
}

\author{
M. V. Ivanov† and P. Schmelcher \\ Theoretische Chemie, Physikalisch-Chemisches Institut, Universität Heidelberg, INF 229, D-69120 Heidelberg, Federal \\ Republic of Germany \\ $\dagger$ †ermanent address: Institute of Precambrian Geology and Geochronology, Russian Academy of Sciences, Nab. Makarova 2, \\ St. Petersburg 199034, Russia
}

(October 24, 2018)

The ground and a few excited states of the beryllium atom in external uniform magnetic fields are calculated by means of our 2D mesh Hartree-Fock method for field strengths ranging from zero up to $2.35 \cdot 10^{9} \mathrm{~T}$. With changing field strength the ground state of the Be atom undergoes three transitions involving four different electronic configurations which belong to three groups with different spin projections $S_{z}=0,-1,-2$. For weak fields the ground state configuration arises from the $1 s^{2} 2 s^{2}, S_{z}=0$ configuration. With increasing field strength the ground state evolves into the two $S_{z}=-1$ configurations $1 s^{2} 2 s 2 p_{-1}$ and $1 s^{2} 2 p_{-1} 3 d_{-2}$, followed by the fully spin polarised $S_{z}=-2$ configuration $1 s 2 p_{-1} 3 d_{-2} 4 f_{-3}$. The latter configuration forms the ground state of the beryllium atom in the high field regime $\gamma>4.567$. The analogous calculations for the $\mathrm{Be}^{+}$ion provide the sequence of the three following ground state configurations: $1 s^{2} 2 s$ and $1 s^{2} 2 p_{-1}\left(S_{z}=-1 / 2\right)$ and $1 s 2 p_{-1} 3 d_{-2}\left(S_{z}=-3 / 2\right)$.

\section{INTRODUCTION}

The behaviour and properties of atoms in strong magnetic fields is a subject of increasing interest. This is motivated by the astrophysical discovery of strong fields on white dwarfs and neutron stars [1 3 . On the other hand the competition of the diamagnetic and Coulomb interaction causes a rich variety of complex properties which are, of course, also of interest on their own.

For a long time the investigations in the literature focused on the hydrogen atom (for a list of references see, for example, 专团). As a result of the corresponding investigations the absorption features of certain magnetic white dwarfs could be understood in detail and a modelling of their atmospheres was possible (see ref. [8] for a review up to 1994 and [9] for more recent references). Detailed spectroscopic calculations were carried out recently for the helium atom in strong magnetic fields [10]. These calculations allow to identify spectra of other, namely helium-rich objects, including the prominent white dwarf GD229 [11. Recently a number of new magnetic white dwarfs have been found whose spectra are still unexplained (see, e.g., Reimers et al [12] in the course of the Hamburg ESO survey).

Investigations on the electronic structure in the presence of a magnetic field appear to be quite complicated due to the intricate geometry of this quantum problem. For the hydrogen atom the impact of the competing Coulomb and diamagnetic interaction is particularly evident and pronounced in the intermediate regime for which the magnetic and Coulomb forces are comparable. For different electronic degrees of excitation of the atom the intermediate regime is met for different absolute values of the field strength. For the ground state this regime corresponds to field strengths around $\gamma=1$ (for the magnetic field strength as well as for other physical values we use atomic units and, in particular, $\gamma=B / B_{0}, B_{0}$ corresponds to the magnetic field strength $B_{0}=\hbar c / e a_{0}^{2}=2.3505 \cdot 10^{5} \mathrm{~T}$ ). Both early [13,14] and more recent works 4,15 on the hydrogen atom have used different approaches for relatively weak fields (the Coulomb force prevails over the magnetic force) and for very strong fields (the Coulomb force can be considered as weak in comparison with the magnetic forces which is the so-called adiabatic regime). A powerful method to obtain comprehensive results on low-lying energy levels of the hydrogen atom in particular in the intermediate regime is provided by mesh methods [5]. For atoms with several electrons there are two decisive factors which enrich the possible changes in the electronic structure with varying field strength compared to the one-electron system. First we have a third competing interaction which is the electron-electron repulsion and second the differ- 
ent electrons feel very different Coulomb forces, i.e. possess different one particle energies, and consequently the regime of the intermediate field strengths appears to be the sum of the intermediate regimes for the separate electrons.

Opposite to the hydrogen atom the wavefunctions of the multi-electron atoms change their symmetries with increasing field strength. It is well known that the singlet zero-field ground state of the helium atom $\left(1 s^{2}\right.$ in the Hartree-Fock language) is replaced in the high-field regime by the triplet fully spin polarised configuration $1 s 2 p_{-1}$. For atoms with more than two electrons the evolution of the ground state within the whole range of field strengths $0 \leq \gamma<+\infty$ includes multiple intermediate configurations besides the zero-field ground state and the ground state corresponding to the high field limit. In view of the above there is a need for further quantum mechanical investigations and data on atoms with more than two electrons in order to understand their electronic structure in strong magnetic fields. Our calculations allowed us to obtain the first conclusive results on the series of ground state configurations for the $\mathrm{Li}$ [16] and $\mathrm{C}$ 17] atoms. These results are substantially different from previously published ones [18]. The ground state electronic configurations of the beryllium atom for $0 \leq \gamma<+\infty$ were not investigated so far. A previous work on the beryllium atom [19] focused on problems associated with the symmetries of the Hartree-Fock wavefunction of the low-field ground state $1 s^{2} 2 s^{2}$ of this atom. For strong fields the $1 s^{2} 2 s^{2}$ state represents a highly excited state and the electronic ground state configuration of Be is, so far, not investigated.

In the current paper we present results of our fully numerical 2D Hartree-Fock mesh calculations of the beryllium atom and $\mathrm{Be}^{+}$ion in magnetic fields and obtain for the first time conclusive results on the structure and energy of the ground state configurations of these systems for arbitrary field strengths.

\section{METHOD}

The computational method applied in the current work coincides with the method described in our works [5, 19, 22, and applied afterwards in [16, 17,23,24]. We solve the electronic Schrödinger equation for the beryllium atom in a magnetic field under the assumption of an infinitely heavy nucleus in the (unrestricted) HartreeFock (HF) approximation. The solution is established in the cylindrical coordinate system $(\rho, \phi, z)$ with the $z$-axis oriented along the magnetic field. We prescribe to each electron a definite value of the magnetic quantum number $m_{\mu}$. Each one-electron wave function $\Psi_{\mu}$ depends on the variables $\phi$ and $(\rho, z)$

$$
\Psi_{\mu}(\rho, \phi, z)=(2 \pi)^{-1 / 2} e^{-i m_{\mu} \phi} \psi_{\mu}(z, \rho)
$$

where $\mu$ indicates the numbering of the electrons. The resulting partial differential equations for $\psi_{\mu}(z, \rho)$ and the formulae for the Coulomb and exchange potentials have been presented in ref. [21]. These equations as well as the Poisson equations for inter-electronic Coulomb and exchange potentials are solved by means of the fully numerical mesh method described in refs. [5,21]. The finitedifference solution of the Poisson equations on sets of nodes coinciding with those of the Hartree-Fock equations turns out to be possible due to a special form of uniform meshes used in the present calculations and in refs. 16, 17, 19. Details and discussion on these meshes are presented in ref. 25].

Our mesh approach is flexible enough to yield precise results for arbitrary field strengths. Some minor decrease of the precision appears for electronic configurations with big differences in the spatial distribution of the electronic density for different electrons. This results in big differences with respect to the spatial extension of the density distribution for different electrons. This situation is more typical for the electronic configurations which do not represent the ground state at the corresponding fields (e.g. $1 s^{2} 2 s^{2}$ at very strong fields or $1 s 2 p_{-1} 3 d_{-2} 4 f_{-3}$ in the weak field regime). The precision of our results depends, of course, on the number of mesh nodes and can be always improved in calculations with denser meshes. Most of the present calculations are carried out on sequences of meshes with the maximal number of nodes being $80 \times 80$.

Along with the numerical solution of the Schrödinger equation the key element for solving the problem of the ground state electronic configurations is a proper choice of the configurations, which could potentially be the ground state ones. An example of solving this problem is presented in [17]. In that work we have developed a 
strategy which enables one to reduce the set of possible ground state configurations which are then subject to a following numerical investigation. This removes the risk of missing some ground state configurations due to the limited possibilities of performing numerical investigations. With increasing number of electrons the number of configurations which cannot a priori be excluded from becoming the ground state increase rapidly. A comprehensive numerical investigation of all these configurations is, in general, not feasible. The above-mentioned strategy to exclude certain configurations is therefore highly desirable. It is based on a combination of qualitative theoretical arguments and numerical calculations of the energies of electronic configurations. As a first step the set of electronic configurations has to be separated into several groups according to their spin projections $S_{z}$. The following considerations have to be carried out in each subset separately, and are certainly more transparent by starting with the limit of infinite strong fields and analysing the electronic configurations with decreasing field strengths. The qualitative theoretical considerations mentioned above are based on the geometry of the spatial part of the wavefunction and enable one to determine the ground state for the high-field limit as well as several candidates for the ground state configuration with decreasing field strength. The numerical calculations then enable us to decide which of these candidates becomes the actual first intermediate ground state and yields the transition field strength. The knowledge of the first intermediate ground state allows us to repeat the qualitative considerations for the second intermediate ground state to obtain a list of candidates which is then investigated by means of numerical calculations. Repeating this procedure one can determine the full sequence of the ground state configurations for each subset $S_{z}$ and finally the sequence of ground state configurations for the physical system.

\section{GROUND STATE ELECTRONIC CONFIGURATIONS FOR $\gamma=0$ AND $\gamma \rightarrow \infty$}

In this section we provide some helpful qualitative considerations on the problem of the atomic multi-electron ground states particularly in the limit of strong magnetic fields.

For the case $\gamma=0$ the ground state configuration of the beryllium atom can be characterised as $1 s^{2} 2 s^{2}$. This notation has a literal meaning when considering the atom in the framework of the restricted Hartree-Fock approach. The latter is an approximation of limited quality in describing the beryllium atom as it was shown in many fully correlated calculations both for the field-free Be atom [26,27 and for its polarizabilities in electric fields [28,29]. It was pointed out in these works that the Be atom is a strongly correlated system and that the HF ground state wavefunction (i. e. the spherically symmetric $1 s^{2} 2 s^{2}$ ) is not a very accurate zeroth-order wavefunction, especially for calculations of electric polarizabilities. This is due to a significant contribution of the $1 s^{2} 2 p^{2}$ configuration to the ground state wave function. The latter configuration is evidently a non-spherical one. This fact is in agreement with results of ref. 19] where the fully numerical 2D unrestricted Hartree-Fock approach provides the $2 s^{2}$ shell stretched along the $z$ axis even for $\gamma=0$. In terms of spherical functions it is natural to describe this geometry of the $2 s^{2}$ shell as a mixture of $2 s$ and $2 p_{0}$ functions. We remark that the $s, p, d \ldots$ orbital notation both for $\gamma=0$ and $\gamma \neq 0$ is based on the behaviour of the wave functions in the vicinity of the origin and on the topology of the nodal surfaces, but does not imply any detailed geometry or certain values of the orbital moment $l$.

It is evident that the field-free ground state of the beryllium atom remains the ground state only for relatively weak fields. The set of one-electron wave functions constituting the $\mathrm{HF}$ ground state for the opposite case of extremely strong magnetic fields can be determined as follows. The nuclear attraction energies and HF potentials (which determine the motion along $z$ axis) are small for large $\gamma$ in comparison to the interaction energies with the magnetic field (which determines the motion perpendicular to the magnetic field and is responsible for the Landau zonal structure of the spectrum). Thus, all the one-electron wavefunctions must correspond to the lowest Landau zones, i.e. the magnetic quantum numbers $m_{\mu}$ are not postive for all the electrons $m_{\mu} \leq 0$, and the system must be fully spin-polarised, i.e. $s_{z \mu}=-\frac{1}{2}$. For the Coulomb central field the one-electron levels form (as $B \rightarrow \infty)$ quasi 1D Coulomb series with the binding en- 
ergy $\epsilon_{B}=\frac{1}{2 n_{z}^{2}}$ for $n_{z}>0$ and $\epsilon_{B} \rightarrow \infty$ for $n_{z}=0$, where $n_{z}$ is the number of nodal surfaces of the wave function with respect to the $z$ axis. The binding energy of a separate electron has the form

$$
\epsilon_{\mathrm{B}}=\left(m+|m|+2 s_{z}+1\right) \gamma / 2-\epsilon
$$

where $\epsilon$ is the energy of the electron.

When considering the case $\gamma \rightarrow \infty$ it is evident, that the wave functions with $n_{z}=0$ have to be chosen for the ground state configuration. Furthermore starting with the energetically lowest one particle level the electrons occupy according to the above arguments orbitals with increasing absolute value of the magnetic quantum number $m_{\mu}$. Consequently the ground state of the beryllium atom must be given by the fully spin-polarised configuration $1 s 2 p_{-1} 3 d_{-2} 4 f_{-3}$. In our notation of the electronic configurations we assume in the following that all paired electrons, like for example the $1 s^{2}$ part of a configuration, are of course in a spin up and spin down orbital, respectively, whereas all unpaired electrons possess a negative projection of the spin onto the magnetic field direction. On a qualitative level the configuration $1 s 2 p_{-1} 3 d_{-2} 4 f_{-3}$ is not very different from similar electronic configurations for other atoms (see ref. 24]). This is a manifestation of the simplification of the picture of atomic properties in the limit $\gamma \rightarrow \infty$ where a linear sequence of electronic configurations replaces the periodic table of elements of the field-free case.

The problem of the configurations of the ground state for the intermediate field region cannot be solved without doing explicit calculations combined with some qualitative considerations in order to extract the relevant configurations.

\section{GROUND STATE ELECTRONIC CONFIGURATIONS FOR ARBITRARY FIELD STRENGTHS}

In order to determine the ground state electronic configurations of the beryllium atom we employ here the strategy introduced in ref. [17] where the carbon atom has been investigated. First of all, we divide the possible ground state configurations into three groups according to their total spin projection $S_{z}$ : the $S_{z}=0$ group (lowfield ground state configurations), the intermediate group $S_{z}=-1$ and the $S_{z}=-2$ group (the high-field ground state configurations). This grouping is required for the following qualitative considerations which are based on the geometry of the spatial parts of the one-electron wave functions. In the course of this discussion it is expedient to treat local ground states for each $S_{z}$ subset (i.e. the lowest states with a certain $S_{z}$ value) along with the global ground state of the atom as a whole. For each value of the magnetic field strength one of these local ground states is the global ground state of the atom.

According to the general arguments presented in the previous section we know that the ground state configuration of the beryllium atom in the high field limit must be the fully spin-polarised state $1 s 2 p_{-1} 3 d_{-2} 4 f_{-3}$. The question of the ground state configurations at intermediate fields cannot be solved without performing explicit electronic structure calculations. On the other hand, the a priori set of possible intermediate ground state configurations increases enormously with increasing number of electrons and is rather large already for the beryllium atom. Some qualitative considerations are therefore needed in order to exclude certain configurations as possible ground state configurations thereby reducing the number of candidates for which explicit calculations have to be performed. As mentioned in the previous section the optimal strategy hereby consists of the repeated procedure of determining neighbouring ground state configurations with increasing (or decreasing) magnetic field strength using both qualitative arguments as well as the results of the calculations for concrete configurations.

The total energies for the considered states and particularly of those states which become the global ground state of the atom for some regime of the field strength are illustrated in figure 1 . In the following paragraphs we describe our sequence of selection procedure and calculations for the candidates of the electronic ground state configurations.

Due to the simplicity of the ground state electronic configurations of atoms in the limit $\gamma \rightarrow \infty$ it is natural to start the consideration for $\gamma \neq 0$ with the high-field ground state and then consider other possible candidates in question for the electronic ground state for $S_{z}=-2$ 
(see figure 1) with decreasing field strength. The consideration of the high-field (i.e. the fully spin-polarised) regime was carried out in ref. 24 and for this case (i.e. $S_{z}=-2$ for beryllium) we repeat this consideration in more detail. In particular, we have found in ref. 24] that the beryllium atom, opposite to the carbon and heavier elements has only one fully spin-polarised ground state configuration.

All the one electron wave functions of the high-field ground state $1 s 2 p_{-1} 3 d_{-2} 4 f_{-3}$ possess no nodal surfaces crossing the $z$-axis and occupy the energetically lowest orbitals with magnetic quantum numbers ranging from $m=0$ down to $m=-3$. The $4 f_{-3}$ orbital possesses the smallest binding energy of all orbitals constituting the high-field ground state. Its binding energy decreases rapidly with decreasing field strength. Thus, we can expect that the first crossover of ground state configurations happens due to a change of the $4 f_{-3}$ orbital into one possessing a higher binding energy at the corresponding lowered field strength. One may think that the first transition while decreasing the magnetic field strength will involve a transition from an orbital possessing $n_{z}=0$ to one for $n_{z}=1$. The energetically lowest available one particle state with $n_{z}=1$ is the $2 p_{0}$ orbital. Another possible orbital into which the $4 f_{-3}$ wave function could evolve is the $2 s$ state. For the hydrogen atom or hydrogen-like ions in a magnetic field the $2 p_{0}$ is stronger bound than the $2 s$ orbital. On the other hand, owing to the electron screening in multi-electron atoms in field-free space the $2 s$ orbital tends to be more tightly bound than the $2 p_{0}$ orbital. Thus, two states i.e. $1 s 2 p_{0} 2 p_{-1} 3 d_{-2}$ and $1 s 2 s 2 p_{-1} 3 d_{-2}$ are candidates for becoming the ground state in the $S_{z}=-2$ set when we lower the field strength coming from the high field situation. The numerical calculations show that the first crossover of the $S_{z}=-2$ subset takes place between the $1 s 2 p_{-1} 3 d_{-2} 4 f_{-3}$ and $1 s 2 p_{0} 2 p_{-1} 3 d_{-2}$ configurations (figure 1). On the other hand, the calculations show that even earlier (i.e. at higher magnetic field strengths) the global ground state acquires the total spin $S_{z}=-1$ due to a crossover of the energy curve of the $1 s 2 p_{-1} 3 d_{-2} 4 f_{-3}$ configuration with that of the configuration $1 s^{2} 2 p_{-1} 3 d_{-2}$ (which is the local ground state for the $S_{z}=-1$ subset in the high-field limit). For the fields below this point $\gamma=4.567$ the ground state electronic configurations of the beryllium atom belong to the subset $S_{z}=-1$. This means that the beryllium atom has only one fully spin polarised ground state configuration (as mentioned above).

The electronic configurations $1 s^{2} 2 p_{-1} 3 d_{-2}$ and $1 s 2 p_{-1} 3 d_{-2} 4 f_{-3}$ differ by the replacement of the spin down $4 f_{-3}$ orbital through the spin up $1 s$ orbital and according to the argumentation presented in the previous section the $1 s^{2} 2 p_{-1} 3 d_{-2}$ represents the local ground state configuration for the subset $S_{z}=-1$ in the limit $\gamma \rightarrow \infty$. Analogous arguments to that presented in the previous paragraph provide the conclusion, that in the process of decreasing field strength the $1 s^{2} 2 p_{-1} 3 d_{-2}$ ground state electronic configuration can be replaced either by the $1 s^{2} 2 s 2 p_{-1}$ or by the $1 s^{2} 2 p_{0} 2 p_{-1}$ configuration. The numerical calculations show, that the curve $E_{1 s^{2} 2 s 2 p_{-1}}(\gamma)$ intersects the curve $E_{1 s^{2} 2 p_{-1} 3 d_{-2}}(\gamma)$ at a higher magnetic field $(\gamma=0.957)$ than $E_{1 s^{2} 2 p_{0} 2 p_{-1}}(\gamma)$ crosses $E_{1 s^{2} 2 p_{-1} 3 d_{-2}}(\gamma)$. The difference with respect to the order of the local ground state configurations in the subsets $S_{z}=-2$ and $S_{z}=-1$ stems from the difference in the magnetic field strengths characteristic for the crossovers in these subsets. At moderate field strengths $\left(S_{z}=-1\right)$ the influence of the Coulomb fields of the nucleus and electrons prevails over the influence of the magnetic field and make the energy of the $2 s$ orbital lower than that of the $2 p_{0}$ orbital. On the other hand, at stronger fields characteristic for the subset $S_{z}=-2$ the energies of these orbitals are governed mostly by the magnetic field and, in result, the energy of the $2 p_{0}$ orbital becomes lower than the energy of the $2 s$ orbital.

From our simple qualitative considerations we can conclude, that the configuration $1 s^{2} 2 s 2 p_{-1}$ is the local ground state configuration of the subset $S_{z}=-1$ for the weak field case, i.e. for $\gamma \rightarrow 0$. Indeed, when we construct such a configuration, the first three electrons go to orbitals $1 s$ and $2 s$ forming the $1 s^{2} 2 s$ configuration with $S_{z}=-1 / 2$. The fourth electron must then have the same spin as the $2 s$ orbital electron to obtain the total spin value $S_{z}=-1$. Thus, the lowest orbital which it can occupy is the $2 p_{-1}$. Therefore, there are two local ground state configurations in the subset $S_{z}=-1$ and they both represent the global ground state for some ranges of the magnetic field strengths. 
The necessary considerations for the subset $S_{z}=0$ are quite simple. At $\gamma=0$ and, evidently, for very weak fields the ground state of the beryllium atom has the configuration $1 s^{2} 2 s^{2}$. We can expect, that when increasing the magnetic field strength, the next lowest state with $S_{z}=0$ will be the $1 s^{2} 2 s 2 p_{-1}$ configuration with opposite directions of the spins of the $2 s$ and $2 p_{-1}$ electrons. But both contributions, the Zeeman spin term and the electronic exchange make the energy of this state higher than the energy of the state $1 s^{2} 2 s 2 p_{-1}$ with the parallel orientation of the spins of the $2 s$ and $2 p_{-1}$ electrons (i.e. $\left.S_{z}=-1\right)$ considered above. The calculated energies for these states are presented in figure 1. Thus, the beryllium atom has one ground state electronic configuration $1 s^{2} 2 s^{2}$ with the total spin $z$-projection $S_{z}=0$. This state is the global ground state for the magnetic field strengths between $\gamma=0$ and $\gamma=0.0412$. Above this point the ground state configuration is $1 s^{2} 2 s 2 p_{-1}$ with $S_{z}=-1$.

Summarising the results on the ground state configurations of the beryllium atom we can state that depending on the magnetic field strength this atom has four different electronic ground state configurations. For $0 \leq \gamma<0.0412$ the ground state configuration coincides with the field-free ground state configuration $1 s^{2} 2 s^{2}$ which has zero values for the total magnetic quantum number $M$ and spin projection $S_{z}$. The following are two ground state configurations with $S_{z}=-1: 1 s^{2} 2 s 2 p_{-1}$ $(M=-1)$ for $0.0412<\gamma<0.957$ and $1 s^{2} 2 p_{-1} 3 d_{-2}$ $(M=-3)$ for $0.957<\gamma<4.567$. For $\gamma>4.567$ the ground state configuration is $1 s 2 p_{-1} 3 d_{-2} 4 f_{-3}$ with $S_{z}=-2$ and $M=-6$. The complete results of the investigations of the sequence of the ground state configurations of the Be atom are presented in table 1 which contains the critical values of $\gamma$ at which the crossovers of different ground state configurations take place.

The next aim of this section is the corresponding investigation of the ground state configurations of the ion $\mathrm{Be}^{+}$. The field-free ground state of this ion corresponds to the $1 s^{2} 2 s$ configuration $\left(S_{z}=-1 / 2\right.$ and $\left.M=0\right)$. In the opposite case $\gamma \rightarrow \infty$ the ground state is obviously given by the $1 s 2 p_{-1} 3 d_{-2}$ configuration $\left(S_{z}=-3 / 2\right.$ and $\left.M=-3\right)$. Thus, we need to investigate only two different subsets of electronic ground state configurations: $S_{z}=-1 / 2$ and
$S_{z}=-3 / 2$. The energy curves which are necessary for this investigation are presented in figure 2 . The subset $S_{z}=-1 / 2$ contains only two possible ground state configurations $1 s^{2} 2 s$ and $1 s^{2} 2 p_{-1}$. The latter is the local ground state configuration for this subset in the limit $\gamma \rightarrow \infty$. The curves $E_{1 s^{2} 2 s}(\gamma)$ and $E_{1 s^{2} 2 p_{-1}}(\gamma)$ intersect at $\gamma=0.3185$ and above this point $E_{1 s^{2} 2 p_{-1}}<E_{1 s^{2} 2 s}$. In the subset $S_{z}=-3 / 2$ we have to consider the configurations $1 s 2 p_{0} 2 p_{-1}$ and $1 s 2 s 2 p_{-1}$ along with the high-field ground state configuration $1 s 2 p_{-1} 3 d_{-2}$. But the numerical calculations show that the energies of both $1 s 2 p_{0} 2 p_{-1}$ and $1 s 2 s 2 p_{-1}$ lie above the energy of the $1 s 2 p_{-1} 3 d_{-2}$ configuration at the intersection point $(\gamma=4.501)$ between $E_{1 s 2 p_{-1} 3 d_{-2}}(\gamma)$ and $E_{1 s^{2} 2 p_{-1}}(\gamma)$. Thus, the ion $\mathrm{Be}^{+}$has three different electronic ground state configurations in external magnetic fields: for $0 \leq \gamma<0.3185$ it is $1 s^{2} 2 s$ $\left(S_{z}=-1 / 2\right.$ and $M=0$ ), then for $0.38483<\gamma<4.501$ it is $1 s^{2} 2 p_{-1}\left(S_{z}=-1 / 2\right.$ and $\left.M=-1\right)$ and for all the values $\gamma>4.501$ the ground state configuration is $1 s 2 p_{-1} 3 d_{-2}\left(S_{z}=-3 / 2\right.$ and $\left.M=-3\right)$. These results are summarised in table [1]. The set of the electronic ground state configurations for the $\mathrm{Be}^{+}$ion appears to be qualitatively the same as for the lithium atom [16]. The field strengths for the corresponding transition points are roughly two times higher for the $\mathrm{Be}^{+}$ion than for the $\mathrm{Li}$ atom.

\section{SELECTED QUANTITATIVE ASPECTS}

In tables III and IV we present the total energies of the four ground state electronic configurations of the beryllium atom and the three ground state electronic configurations of the ion $\mathrm{Be}^{+}$, respectively. These data cover a very broad range of the field strengths from $\gamma=0$ and very weak magnetic fields starting with $\gamma=0.001$ up to extremely strong fields $\gamma=10000$. The latter value of the field strength can be considered as a rough limit of applicability of the non-relativistic quantum equations to the problem (see below). The corresponding data on the $\mathrm{Be}^{+}$ion can be found in tables II and IV.

So far there exist three works which should be mentioned in the context of the problem of the beryllium atom in strong magnetic fields. Ref. [19] deals with the $1 s^{2} 2 s^{2}$ state of this atom in fields $0 \leq \gamma \leq 1000$ and 
ref. [24] investigates the ground state energies of atoms with nuclear charge number $Z \leq 10$ in the high-field, i.e. fully spin polarised regime. Both these works contain calculations carried out by the method used in the current work and do not represent a basis for comparison. The comparison of our results with an adiabatic HartreeFock calculation of atoms with $Z \leq 10$ [30] is presented in [24] and we can briefly summarise this comparison for two values of the magnetic field strengths: for $B_{12}=0.1$ (i.e. $B=0.1 \times 10^{12} \mathrm{G}$ ) our result is $E=-0.89833 \mathrm{keV}$ whereas ref. [30] yields $E=-0.846 \mathrm{keV}$; for $B_{12}=5$ (i.e. $B=5 \times 10^{12} \mathrm{G}$ ) our result is $E=-3.61033 \mathrm{keV}$, whereas ref. [30] yields $E=-3.5840 \mathrm{keV}$. This comparison allows us to draw the conclusion of a relatively low precision of the adiabatic approximation for multi-electron atoms even for relatively high magnetic fields.

In figure 3 we present the ionization energy $E_{\text {ion }}$ of the beryllium atom depending on the magnetic field strength. This continuous dependence is divided into six parts corresponding to different pairs of the ground state configurations of the $\mathrm{Be}$ atom and $\mathrm{Be}^{+}$ion involved into the ionization energy. The five vertical dotted lines in figure 3 mark the boundaries of these sections. The alteration of the sections of growing and decreasing ionization energy originates from different dependencies of the total energies of the $\mathrm{Be}$ and $\mathrm{Be}^{+}$on the magnetic field strength for different pairs of the ground state configurations of these two systems. One can see the sharp decrease of the ionization energy between the crossovers (4) and (5). This behaviour is due to the fact that $E_{\text {ion }}$ is determined in this section by the rapidly decreasing total energy of the state $1 s 2 p_{-1} 3 d_{-2}$ of the $\mathrm{Be}^{+}$ion (figure 2 ) and by the energy of the Be atom in the state $1 s^{2} 2 p_{-1} 3 d_{-2}$ which is very weakly dependent on the field strength (figure 1 ). Another remarkable feature of the curve $E_{\text {ion }}(\gamma)$ is its behaviour in the range of field strengths between the transitions (2) and (3). The ionization energy in this region contains a very shallow maximum and in the whole section it is almost independent on the magnetic field. Thus, the ionization energy is stationary in this regime of field strengths $\gamma=0.3-0.5$ a.u. typical for many magnetic white dwarfs [8].

The above-discussed properties are based on the behaviour of the total energy of the $\mathrm{Be}$ atom and $\mathrm{Be}^{+}$ion.
On the other hand, the behaviour of the wavefunctions and many intrinsic characteristics of atoms in external magnetic fields are associated not with the total energy, but with the binding energies of separate electrons (2) and the total binding energy of the system

$$
E_{\mathrm{B}}=\sum_{\mu=1}^{N}\left(m_{\mu}+\left|m_{\mu}\right|+2 s_{z \mu}+1\right) \gamma / 2-E
$$

where $N$ is the number of electrons. The binding energies of the ground state electronic configurations of $\mathrm{Be}$ and $\mathrm{Be}^{+}$depending on the magnetic field strength are presented in figures 4 and 5 . These dependencies at very strong magnetic fields may illustrate our considerations of the previous sections. One can see in figure 4 that the high-field ground state $1 s 2 p_{-1} 3 d_{-2} 4 f_{-3}$ is not the most tightly bound state of the beryllium atom. For all the values of the magnetic fields considered in this paper its binding energy is lower than that of states $1 s^{2} 2 s 2 p_{-1}$ and $1 s^{2} 2 p_{-1} 3 d_{-2}$ and for $\gamma<100$ it is lower than $E_{\mathrm{B} 1 s^{2} 2 s^{2}}$. The latter circumstance can be easily explained by the fact that the $1 s^{2} 2 s^{2}$ configuration contains two tightly bound orbitals $1 s$ whereas the $1 s 2 p_{-1} 3 d_{-2} 4 f_{-3}$ possess only one such orbital. However, with increasing magnetic field strengths the contribution of the group $2 p_{-1} 3 d_{-2} 4 f_{-3}$ to the binding energy turns out to be larger than that of the $1 s 2 s^{2}$ group. Analogously we can expect $E_{\mathrm{B} 1 s 2 p_{-1} 3 d_{-2} 4 f_{-3}}>E_{\mathrm{B} 1 s^{2} 2 s 2 p_{-1}}$ at some very large fields $\gamma>10000$. On the other hand, it is evident that the state $1 s 2 p_{-1} 3 d_{-2} 4 f_{-3}$ must be less bound than $1 s^{2} 2 p_{-1} 3 d_{-2}$ because both these configurations are constructed of orbitals with binding energies, logarithmically increasing as $\gamma \rightarrow \infty$, but the $1 s^{2} 2 p_{-1} 3 d_{-2}$ contains an additional $1 s$ orbital, which is more tightly bound than $4 f_{-3}$ at arbitrary field strengths. The plot for the $\mathrm{Be}^{+}$ion (figure 5) illustrates the same features and one can see in this figure nearly parallel curves $E_{\mathrm{B} 1 s^{2} 2 p_{-1}}(\gamma)$ and $E_{\mathrm{B} 1 s 2 p_{-1} 3 d_{-2}}(\gamma)$ in the strong field regime.

Figures 6 and 7 allow us to add some details to the considerations of the previous section. These figures present spatial distributions of the total electronic densities for the ground state configurations of the beryllium atom and its positive ion, respectively. These pictures allow us to gain insights into the geometry of the distribution of the electronic density in space and in par- 
ticular its dependence on the magnetic quantum number and the total spin. The first pictures in these figures present the distribution of the electronic density for the ground state for $\gamma=0$. The following pictures show the distributions of the electronic densities for values of the field strength which mark the boundaries of the regimes of field strengths belonging to the different ground state configurations. For the high-field ground states we present the distribution of the electronic density at the crossover field strength and at $\gamma=500$. For each configuration the effect of the increasing field strength consists in compressing the electronic distribution towards the $z$ axis. However the crossovers of ground state configurations involve the opposite effect due to the fact that these crossovers are associated with an increase of the total magnetic quantum number $M$.

In the first rows of figures 6 and 7 one can see a dense core of $1 s^{2}$ electrons inside the bold solid line contour and a diffuse distribution of $2 s$ electrons outside this core. The prolate shape of the bold solid line contour in the first plot of the figure $6\left(1 s^{2} 2 s^{2}, \gamma=0\right)$ reflects the non-spherical distribution of the $2 s$ electrons in our calculations or the admixture of the $1 s^{2} 2 p_{0}^{2}$ configuration to the $1 s^{2} 2 s^{2}$ one from the point of view of the multiconfigurational approach [26 29].

Some additional issues concerning the results presented above have to be discussed. First, our HF results do not include the effects of correlation. To take into account the latter would require a multi-configurational approach which goes beyond the scope of the present paper. We, however, do not expect that the correlation energy changes our main conclusions like, for example, the fact of the crossovers with respect to the different ground states configurations. With increasing field strength the effective one particle picture should be an increasingly better description of the wave function and the percentage of the correlation energy should therefore decrease (see ref. [23] for an investigation on this subject). Two other important issues are relativistic effects and effects due to the finite nuclear mass. Both these points are basically important for very high magnetic field strengths and they have been discussed in ref. 24]. For the systems Be and $\mathrm{Be}^{+}$and for most of the field strengths considered here these effects result in minor corrections to the energy.

\section{SUMMARY AND CONCLUSIONS}

We have applied our 2D mesh Hartree-Fock method to the magnetised neutral beryllium atom and beryllium positive ion. The method is flexible enough to yield precise results for arbitrary field strengths and our calculations for the ground and several excited states are performed for magnetic field strengths ranging from zero up to $2.3505 \cdot 10^{9} \mathrm{~T}(\gamma=10000)$. Our considerations focused on the ground states and their crossovers with increasing field strength. The ground state of the beryllium atom undergoes three transitions involving four different electronic configurations. For weak fields up to $\gamma=0.0412$ the ground state arises from the field-free ground state configuration $1 s^{2} 2 s^{2}$ with the total spin $z$-projection $S_{z}=0$. With increasing strength of the field two different electronic configurations with $S_{z}=-1$ consequently become the ground state: $1 s^{2} 2 s 2 p_{-1}$ and $1 s^{2} 2 p_{-1} 3 d_{-2}$. At $\gamma=4.567$ the last crossover of the ground state configurations takes place and for $\gamma>4.567$ the ground state wavefunction is represented by the high-field-limit fully spin polarised configuration $1 s 2 p_{-1} 3 d_{-2} 4 f_{-3}, S_{z}=-2$.

For the ion $\mathrm{Be}^{+}$we obtain three different ground state configurations possessing two values of the spin projection. For fields below $\gamma=0.3185$ the ground state electronic configuration has the spin projection $S_{z}=$ $-1 / 2$, magnetic quantum number $M=0$ and qualitatively coincides with the zero-field ground state configuration $1 s^{2} 2 s$. Between $\gamma=0.3185$ and $\gamma=4.501$ the ground state is represented by another configuration with $S_{z}=-1 / 2$, i.e. $1 s^{2} 2 p_{-1}(M=-1)$. Above the point $\gamma=4.501$ the fully spin polarised high-field-limit configuration $1 s 2 p_{-1} 3 d_{-2}\left(S_{z}=-3 / 2\right)$ is the actual ground state of the $\mathrm{Be}^{+}$ion. Thus, the sequence of electronic ground state configurations for the $\mathrm{Be}^{+}$ion is similar to the sequence for the $\mathrm{Li}$ atom [16]. We present detailed tables of energies of the ground state configurations for Be and $\mathrm{Be}^{+}$.

For $\mathrm{Be}$ and $\mathrm{Be}^{+}$we have presented also the binding energies of the ground state configurations dependent on the magnetic field strength and maps of electronic densities for these configurations. For the Be atom we present its ionization energy dependent on the field strength.

Our investigation represents the first conclusive study 
of the ground state of the beryllium atom and $\mathrm{Be}^{+}$ion for arbitrary field strengths. For the Be atom we have obtained a new sequence of electronic configurations with increasing field strength. This sequence does not coincide with any such sequences obtained previously for other atoms and ions and could not be predicted even qualitatively without detailed calculations. Putting together what we currently know about ground states of atomic systems in strong magnetic fields we can conclude that the $\mathrm{H}, \mathrm{He}, \mathrm{Li}, \mathrm{Be}, \mathrm{C}, \mathrm{He}^{+}, \mathrm{Li}^{+}$and $\mathrm{Be}^{+}$ground states have been identified. For other atoms and multiple series of ions the question about the ground state configurations is still open.

[1] J. P. Ostriker and F. D. A. Hartwick, Astrophys. J. 153, 797 (1968).

[2] J. Trümper, W. Pietsch, C. Reppin, W. Voges, R. Stauben, and E. Kendziorra, Astrophys. J. 219, L105 (1978).

[3] J. D. Landstreet, in Cosmical Magnetism, edited by D. Lynden-Bell (Kluwer, Boston, 1994), p.55.

[4] W. Rösner, G. Wunner, H. Herold, and H. Ruder, J. Phys. B 17, 29 (1984).

[5] M. V. Ivanov, J. Phys. B 21, 447 (1988).

[6] H. Friedrich and D. Wintgen, Phys.Rep. 183, 37 (1989).

[7] Yu. P. Kravchenko, M. A. Liberman, and B. Johansson, Phys.Rev.Lett. 77, 619 (1996).

[8] H. Ruder, G. Wunner, H. Herold and F. Geyer, Atoms in Strong Magnetic Fields, Springer-Verlag 1994.

[9] Atoms and Molecules in Strong External Fields, edited by P. Schmelcher and W. Schweizer, Plenum Press New York and London (1998)

[10] W. Becken, P. Schmelcher, and F.K. Diakonos, J. Phys.
B 32, 1557 (1999).

[11] S. Jordan, P. Schmelcher, W. Becken, and W. Schweizer, Astr.\&Astrophys. 336, 33 (1998).

[12] D. Reimers, S. Jordan, V. Beckmann, N. Christlieb, L. Wisotzki, Astr.\& Astrophys. 337, L13 (1998)

[13] R. H. Garstang, Rep. Prog. Phys. 40, 105 (1977).

[14] J. Simola and J Virtamo, J. Phys. B 11, 3309 (1978).

[15] H. Friedrich, Phys. Rev. A 26, 1827 (1982).

[16] M. V. Ivanov and P. Schmelcher, Phys. Rev. A 57, 3793 (1998).

[17] M. V. Ivanov and P. Schmelcher, Phys. Rev. A 60, 3558 (1999).

[18] M. D. Jones, G. Ortiz, and D. M. Ceperley, Phys. Rev. A. 54, 219 (1996).

[19] M. V. Ivanov, Phys. Lett. A 239, 72 (1998).

[20] M. V. Ivanov, Optics and Spectroscopy 70, 148 (1991).

[21] M. V. Ivanov, J. Phys. B 27, 4513 (1994).

[22] M. V. Ivanov, USSR Comput. Math. \& Math. Phys. 26, 140 (1986).

[23] P. Schmelcher, M. V. Ivanov and W. Becken, Phys. Rev. A 59, 3424 (1999).

[24] M. V. Ivanov and P. Schmelcher, Phys. Rev. A 61, 022505 (2000).

[25] M. V. Ivanov and P. Schmelcher, Advances in Quantum Chemistry, to be published.

[26] K. J. Miller, and K. Ruedenberg, J. Chem. Phys. 48, 3450 (1968).

[27] J. S. Sims and S. Hagstrom, Phys. Rev. A 4, 908 (1971).

[28] G. H. F. Diercksen and A. J. Sadlej, Chem. Phys. 77, 429 (1983).

[29] G. Maroulis and A. J. Thakkar, J. Phys. B: At. Mol. Opt. Phys. 21, 3819 (1988).

[30] D. Neuhauser, S. E. Koonin, and K. Langanke, Phys. Rev. A 33, 2084 (1986); 36, 4163 (1987). 


\section{Figure Captions}

Figure 1. The total energies (in atomic units) of the states of the beryllium atom as functions of the magnetic field strength considered for the determination of the ground state electronic configurations. The field strength is given in units of $\gamma=\left(\frac{B}{B_{0}}\right), B_{0}=\hbar c / e a_{0}^{2}=2.3505 \cdot 10^{5} \mathrm{~T}$.

Figure 2. The total energies (in atomic units) of the states of the beryllium positive ion as functions of the magnetic field strength considered for the determination of the ground state electronic configurations. The field strength is given in units of $\gamma=\left(\frac{B}{B_{0}}\right), B_{0}=\hbar c / e a_{0}^{2}=$ $2.3505 \cdot 10^{5} \mathrm{~T}$.

Figure 3. Be atom ground state ionization energy $E_{I}$. Transition points are marked by broken vertical lines. The sequence of the transitions are (from left to right): 1 . Be: $1 s^{2} 2 s^{2} \longrightarrow 1 s^{2} 2 s 2 p_{-1} ; 2 . \mathrm{Be}^{+}: 1 s^{2} 2 s \longrightarrow$ $1 s^{2} 2 p_{-1}$; 3. Be: $1 s^{2} 2 s 2 p_{-1} \longrightarrow 1 s^{2} 2 p_{-1} 3 d_{-2}$. 4. $\mathrm{Be}^{+}:$ $1 s^{2} 2 p_{-1} \longrightarrow 1 s 2 p_{-1} 3 d_{-2} ; 5$. Be: $1 s^{2} 2 p_{-1} 3 d_{-2} \longrightarrow$ $1 s 2 p_{-1} 3 d_{-2} 4 f_{-3}$. Crossovers (4) and (5) take place at relatively close values of $\gamma$ and are not resolved in the figure.

Figure 4. The binding energies (in atomic units) of the ground state electronic configurations of the Be atom depending on the magnetic field strength. The field strength is given in units of $\gamma=\left(\frac{B}{B_{0}}\right), B_{0}=\hbar c / e a_{0}^{2}=$ $2.3505 \cdot 10^{5} \mathrm{~T}$.

Figure 5. The binding energies (in atomic units) of the ground state electronic configurations of the $\mathrm{Be}^{+}$ ion depending on the magnetic field strength. The field strength is given in units of $\gamma=\left(\frac{B}{B_{0}}\right), B_{0}=\hbar c / e a_{0}^{2}=$ $2.3505 \cdot 10^{5} \mathrm{~T}$.

Figure 6. Contour plots of the total electronic densities for the ground state of the beryllium atom. For neighbouring lines the densities are different by a factor of 2 . The coordinates $z, x$ as well as the corresponding field strengths are given in atomic units. Each row presents plots for a ground state configuration at its lower (left) and upper (right) intersection points. Rows: 1. $1 s^{2} 2 s^{2}$ : $\gamma=0$ and $\gamma=0.0412 ; 2.1 s^{2} 2 s 2 p_{-1}: \gamma=0.0412$ and $\gamma=0.957 ; 3$. $1 s^{2} 2 p_{-1} 3 d_{-2}: \gamma=0.957$ and $\gamma=4.567 ; 4$.
$1 s 2 p_{-1} 3 d_{-2} 4 f_{-3}: \gamma=4.567$ and $\gamma=500$.

Figure 7. Contour plots of the total electronic densities for the ground state of the beryllium positive ion. For neighbouring lines the densities are different by a factor of 2 . The coordinates $z, x$ as well as the corresponding field strengths are given in atomic units. Each row presents plots for a ground state configuration at its lower (left) and upper (right) intersection points. Rows: 1. $1 s^{2} 2 s: \gamma=0$ and $\gamma=0.3185 ; 2.1 s^{2} 2 p_{-1}: \gamma=0.3185$ and $\gamma=4.501 ; 3$. $1 s 2 p_{-1} 3 d_{-2}: \gamma=4.501$ and $\gamma=500$. 
TABLE I. The Hartree-Fock ground state configurations of the beryllium atom in external magnetic fields. The configurations presented in the table are the ground state configurations for $\gamma_{\min } \leq \gamma \leq \gamma_{\max }$. Atomic units are used.

\begin{tabular}{llllrll}
\hline \hline no. & $\gamma_{\min }$ & $\gamma_{\max }$ & The ground state configuration & $M$ & $S_{z}$ & $E\left(\gamma_{\min }\right)$ \\
\hline 1 & 0 & 0.0412 & $1 s^{2} 2 s^{2}$ & 0 & 0 & -14.57336 \\
2 & 0.0412 & 0.957 & $1 s^{2} 2 s 2 p_{-1}$ & -1 & -1 & -14.57098 \\
3 & 0.957 & 4.567 & $1 s^{2} 2 p_{-1} 3 d_{-2}$ & -3 & -1 & -15.13756 \\
4 & 4.567 & $\infty$ & $1 s 2 p_{-1} 3 d_{-2} 4 f_{-3}$ & -6 & -2 & -15.91660 \\
\hline \hline
\end{tabular}

TABLE II. The Hartree-Fock ground state configurations of the $\mathrm{Be}^{+}$ion in external magnetic fields. The configurations presented in the table are the ground state configurations for $\gamma_{\min } \leq \gamma \leq \gamma_{\max }$. Atomic units are used.

\begin{tabular}{llllrll}
\hline \hline no. & $\gamma_{\min }$ & $\gamma_{\max }$ & The ground state configuration & $M$ & $S_{z}$ & $E\left(\gamma_{\min }\right)$ \\
\hline 1 & 0 & 0.3185 & $1 s^{2} 2 s$ & 0 & $-1 / 2$ & -14.27747 \\
2 & 0.3185 & 4.501 & $1 s^{2} 2 p_{-1}$ & -1 & $-1 / 2$ & -14.38602 \\
3 & 4.501 & $\infty$ & $1 s 2 p_{-1} 3 d_{-2}$ & -3 & $-3 / 2$ & -15.01775 \\
\hline \hline
\end{tabular}


TABLE III. The total energies of the ground state configurations of the beryllium atom depending on the magnetic field strength. The figures in parentheses are the labels of the ground state configurations provided in the first column of table I. Atomic units are used.

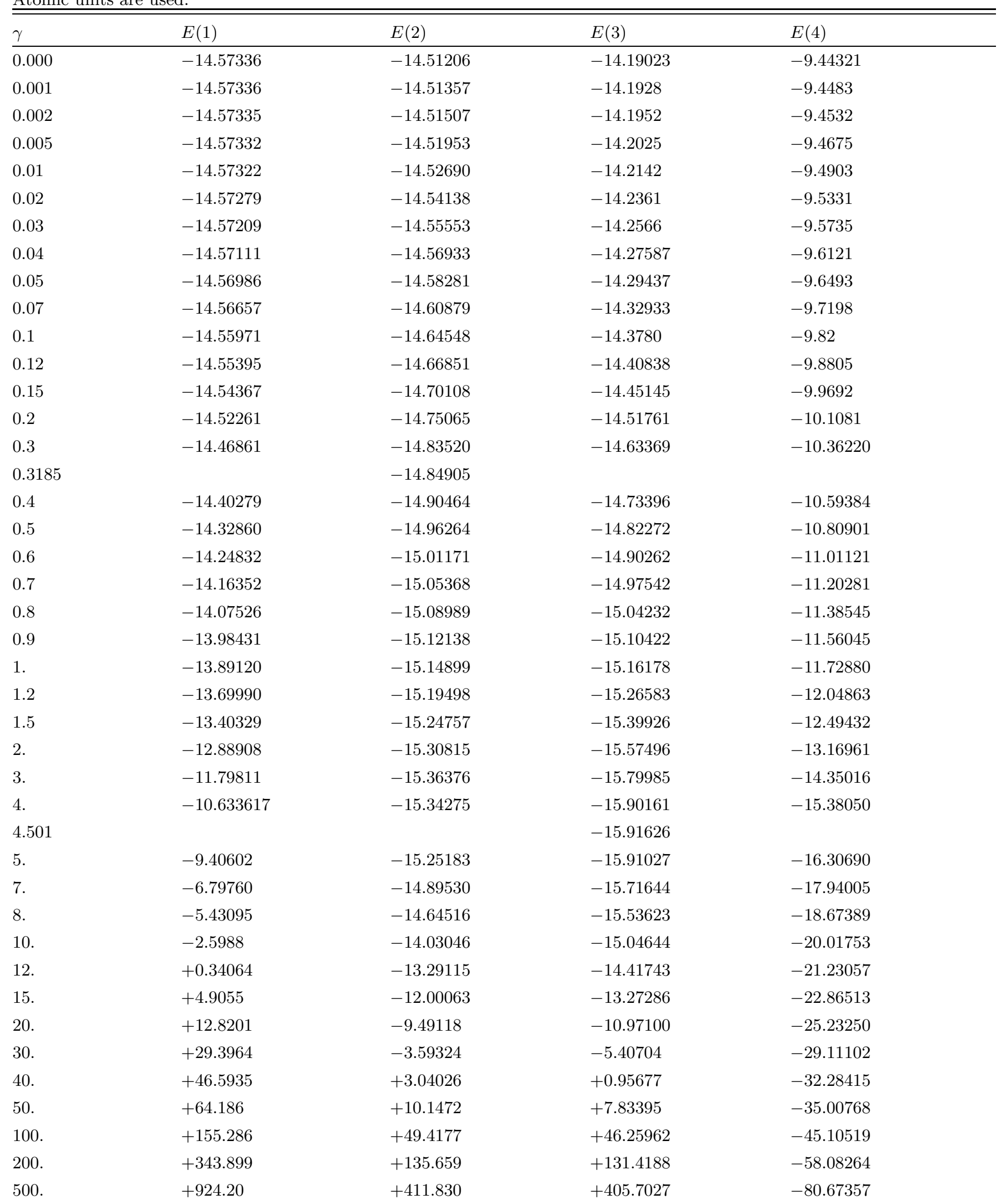




\begin{tabular}{|c|c|c|c|c|}
\hline 1000. & +1905.14 & +888.70 & +880.706 & -102.75480 \\
\hline 2000 & +3881.5 & +1860.40 & +1850.052 & -129.9790 \\
\hline 5000. & & +4813.56 & +4799.35 & -175.2704 \\
\hline 10000. & & +9770.37 & +9752.24 & -217.695 \\
\hline
\end{tabular}


TABLE IV. The total energies of the ground state configurations of the $\mathrm{Be}^{+}$ion depending on the magnetic field strength. The figures in parentheses are the labels of the ground state configurations provided in the first column of table II. Atomic units are used.

\begin{tabular}{|c|c|c|c|}
\hline$\gamma$ & $E(1)$ & $E(2)$ & $E(3)$ \\
\hline 0.001 & -14.27797 & -14.13195 & -9.41358 \\
\hline 0.005 & -14.27995 & -14.13593 & -9.42551 \\
\hline 0.01 & -14.28241 & -14.14087 & -9.44028 \\
\hline 0.03 & -14.29198 & -14.16030 & -9.49791 \\
\hline 0.04 & -14.29659 & -14.16980 & -9.52587 \\
\hline 0.0412 & -14.29714 & & \\
\hline 0.05 & -14.30111 & -14.17916 & -9.55332 \\
\hline 0.07 & -14.30981 & -14.19746 & -9.60670 \\
\hline 0.15 & -14.34047 & -14.26542 & -9.80463 \\
\hline 0.2 & -14.35648 & -14.30406 & -9.91878 \\
\hline 0.3 & -14.38212 & -14.37402 & -10.13144 \\
\hline 0.4 & -14.40046 & -14.43599 & -10.32817 \\
\hline 0.5 & -14.41282 & -14.49163 & -10.51259 \\
\hline 0.6 & -14.42022 & -14.54210 & -10.68705 \\
\hline 0.7 & -14.42350 & -14.58821 & -10.85323 \\
\hline 0.8 & -14.42335 & -14.63059 & -11.01236 \\
\hline 0.9 & -14.42029 & -14.66971 & -11.16540 \\
\hline 4. & -13.83797 & -15.05004 & -14.58615 \\
\hline 4.567 & & & -15.07310 \\
\hline 5. & -13.55019 & -14.96820 & -15.42817 \\
\hline 7. & -12.85647 & -14.61928 & -16.91814 \\
\hline 8. & -12.45821 & -14.37080 & -17.58931 \\
\hline 10. & -11.57652 & -13.75773 & -18.820184 \\
\hline 12. & -10.59993 & -13.01900 & -19.93310 \\
\hline 15. & -8.99386 & -11.72840 & -21.43461 \\
\hline 20. & -6.03364 & -9.217910 & -23.612005 \\
\hline 30. & +0.59244 & -3.31723 & -27.18373 \\
\hline 40. & +7.81557 & +3.31895 & -30.10832 \\
\hline 50. & +15.4261 & +10.42836 & -32.61959 \\
\hline 100. & +56.5516 & +49.70820 & -41.93414 \\
\hline 200. & +145.1649 & +135.95916 & -53.90638 \\
\hline
\end{tabular}




\begin{tabular}{llll}
500. & +425.471 & +412.14745 & -74.73619 \\
1000. & +906.37 & +889.0264 & -95.07513 \\
2000. & +1883.08 & +1860.7100 & -120.11947 \\
5000. & +4844.6 & +4814.005 & -161.7052 \\
10000. & +9809.3 & +9770.643 & -200.5709 \\
\hline \hline
\end{tabular}


Figure 1

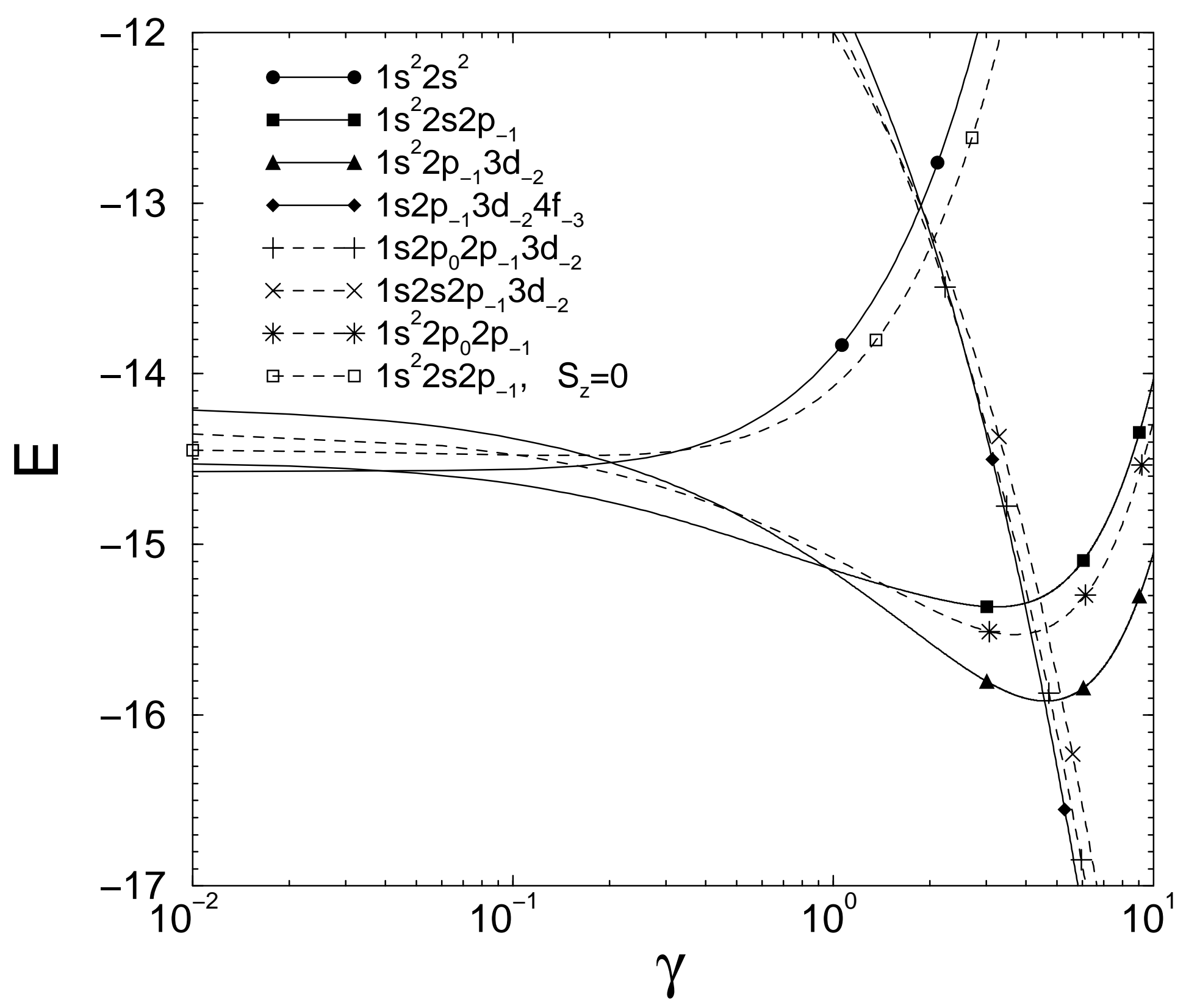


Figure 2

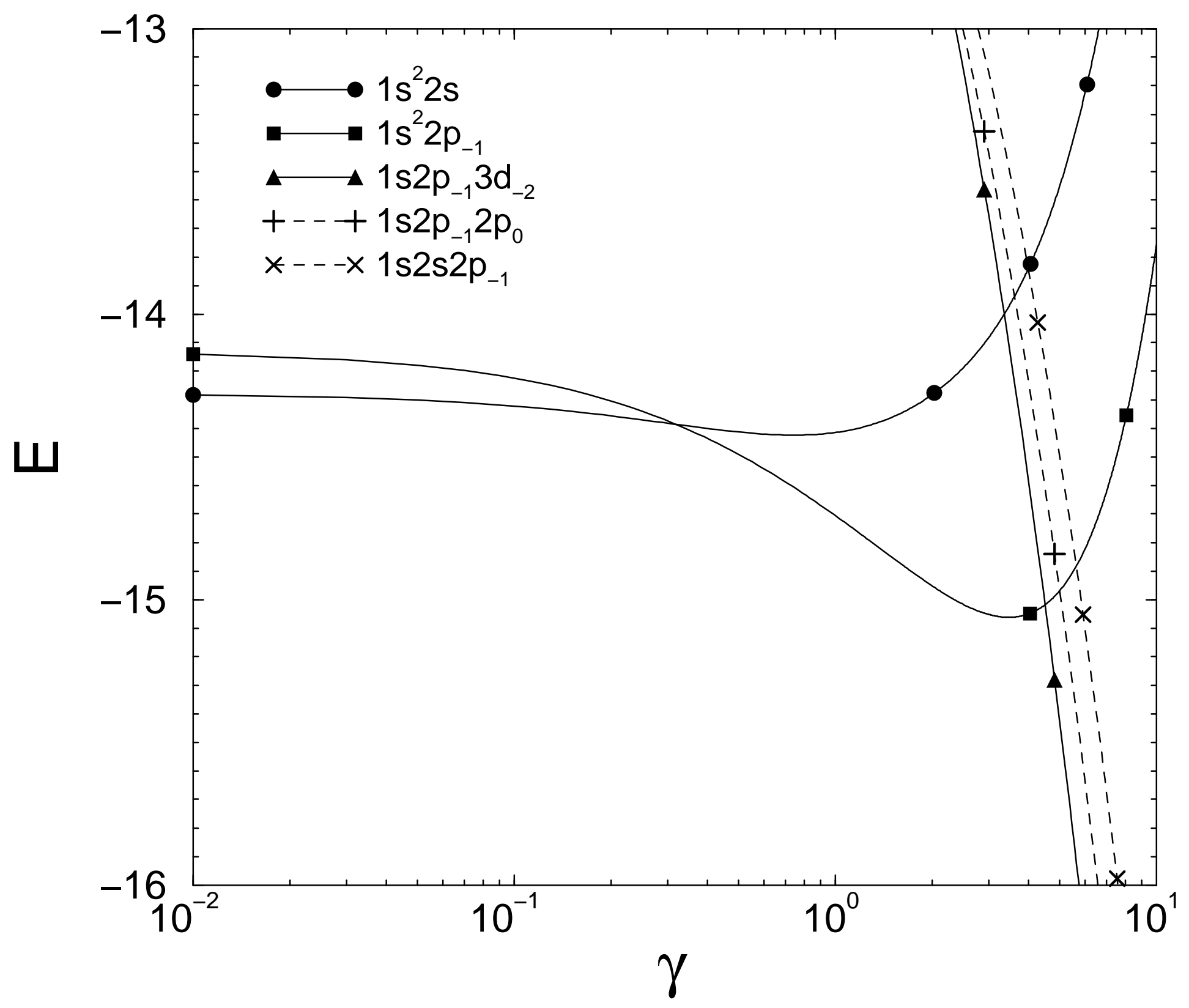


Figure 3

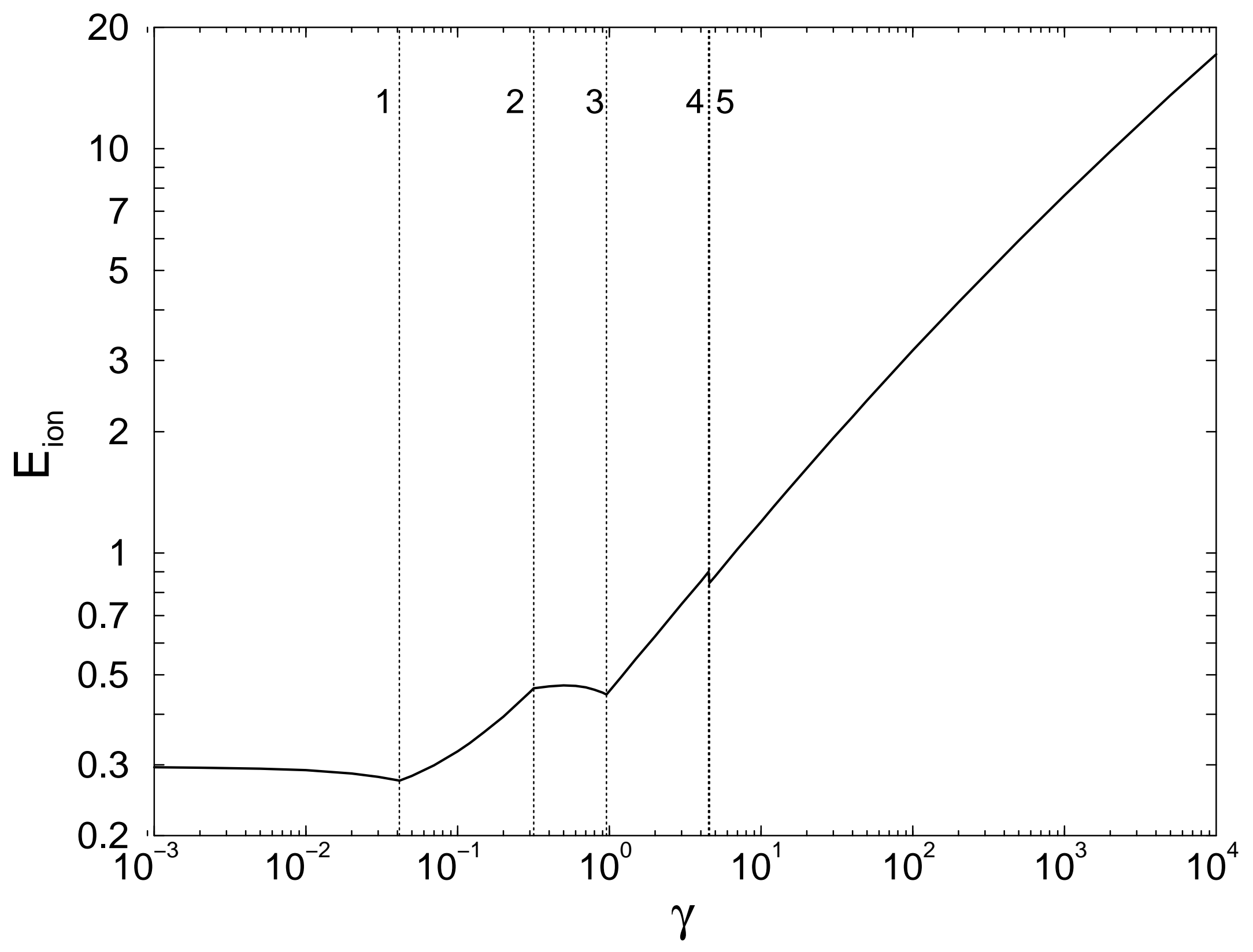


Figure 4

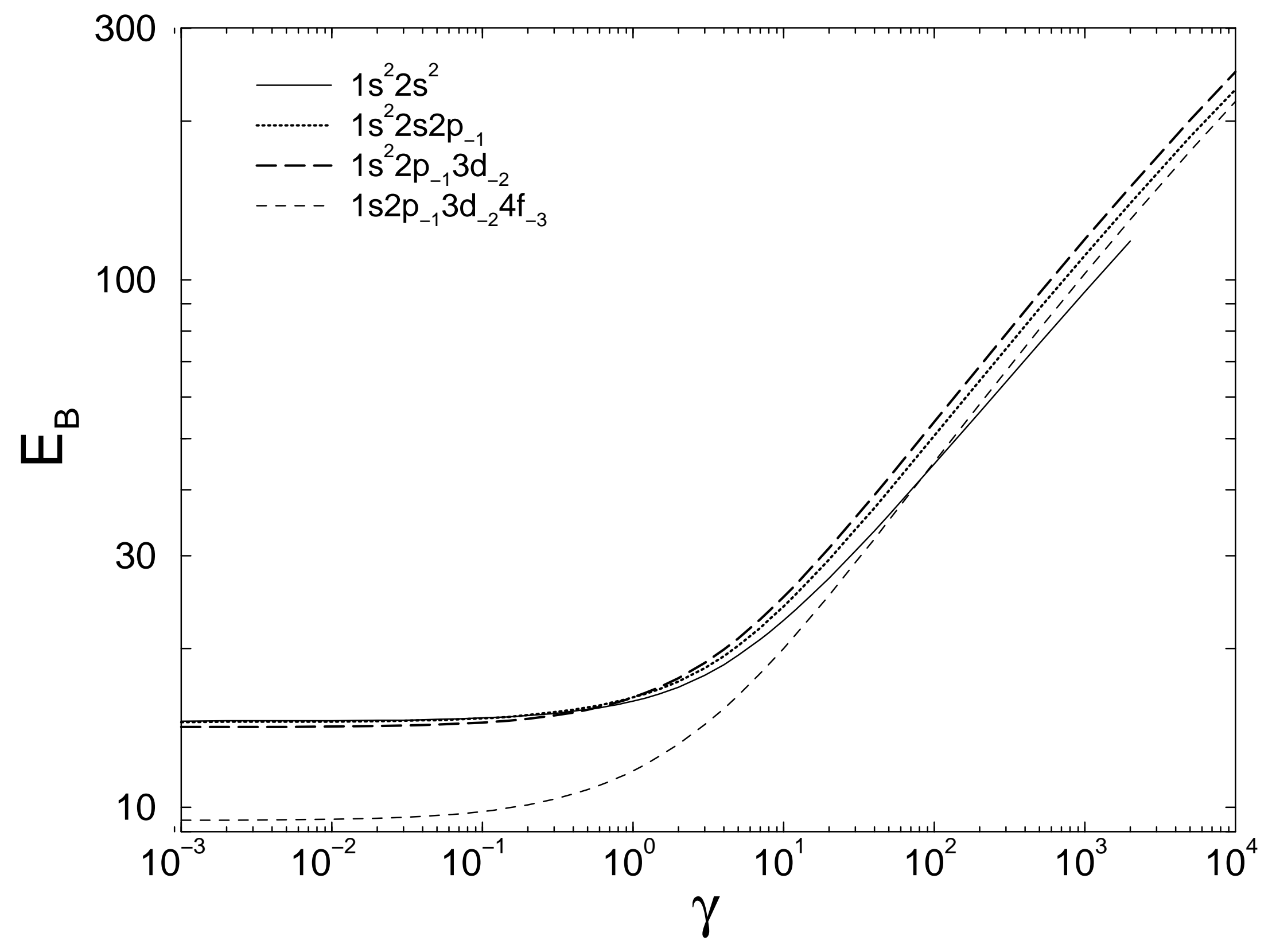


Figure 5

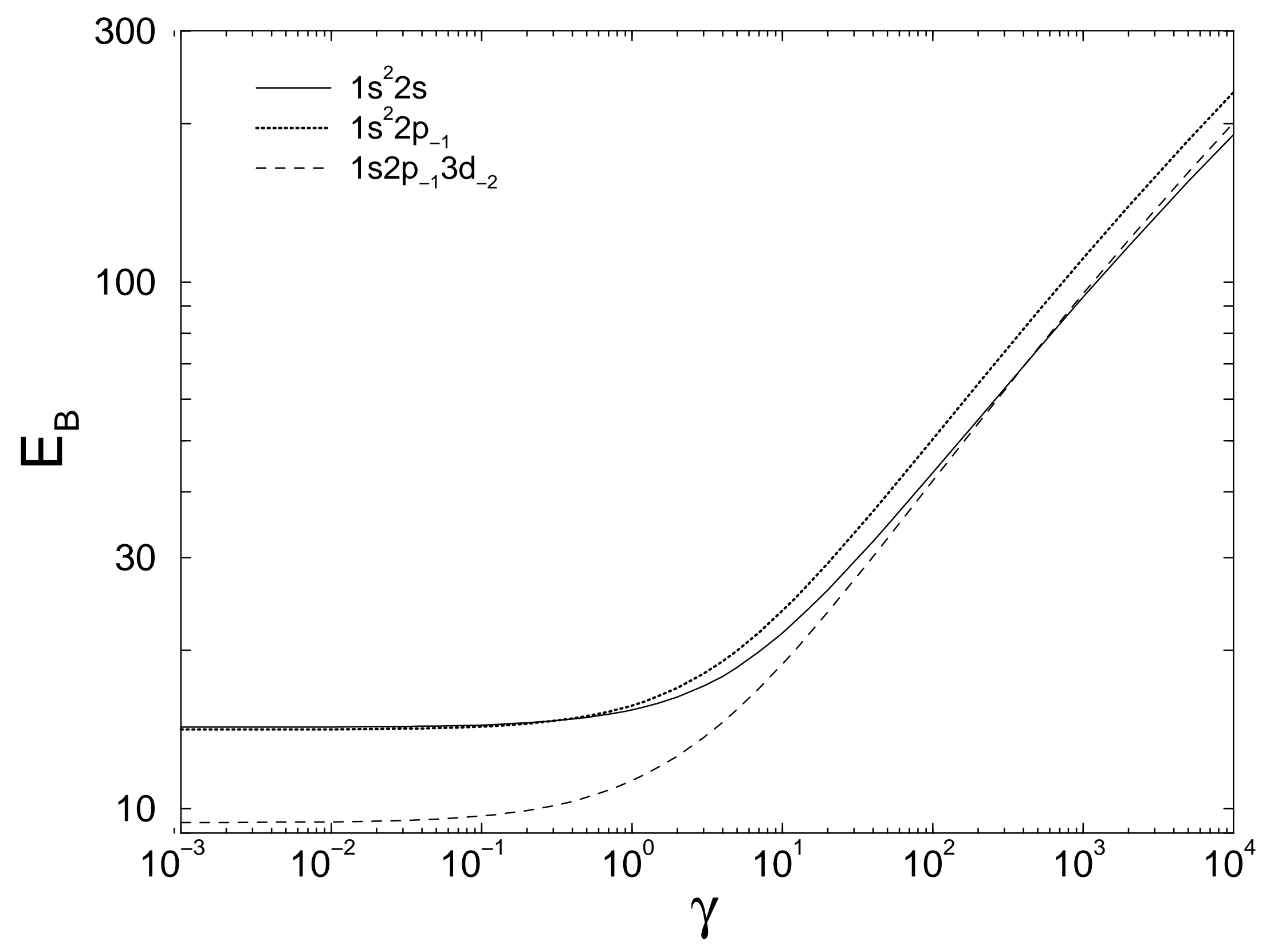



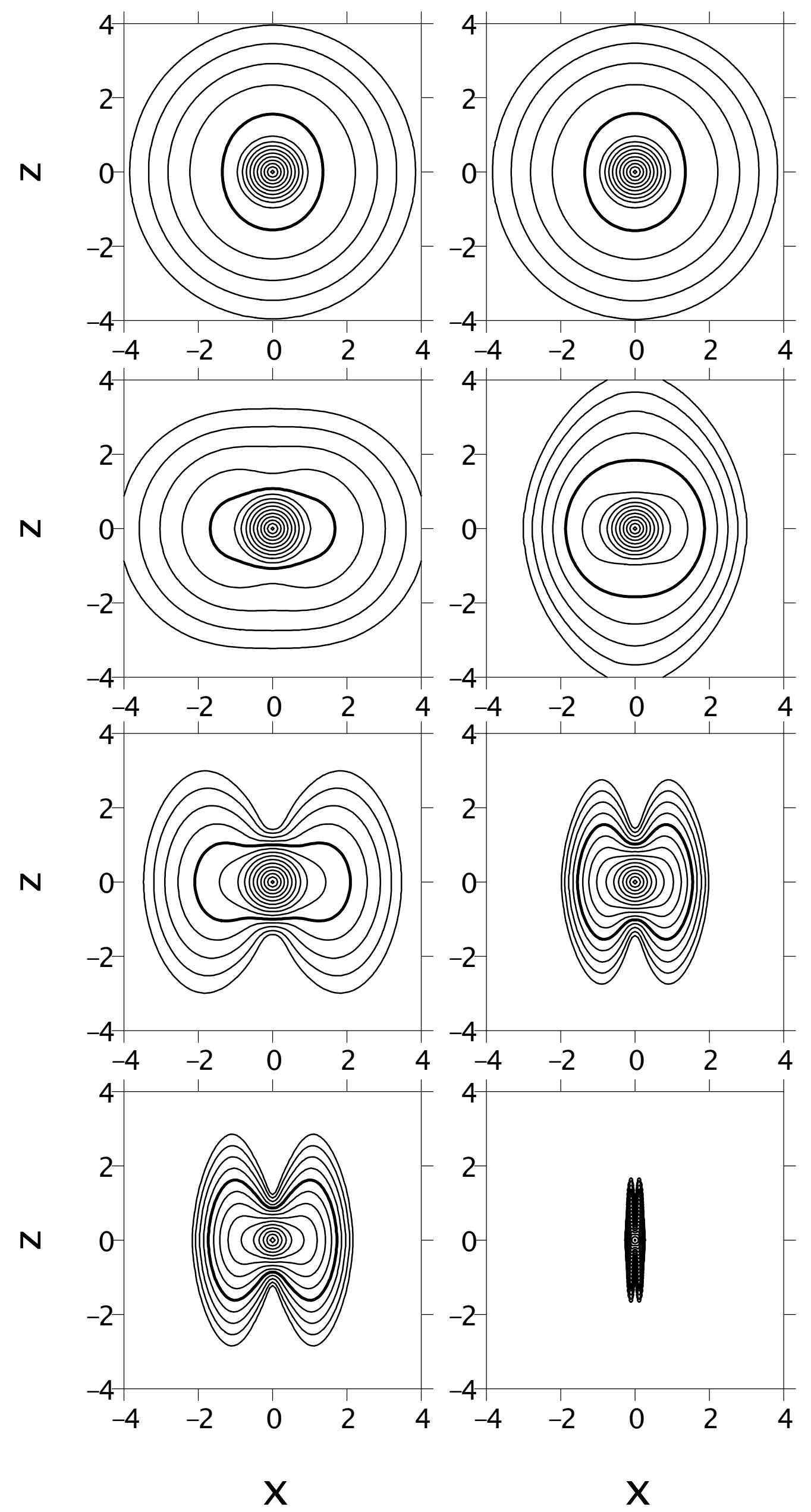

Figure 6 

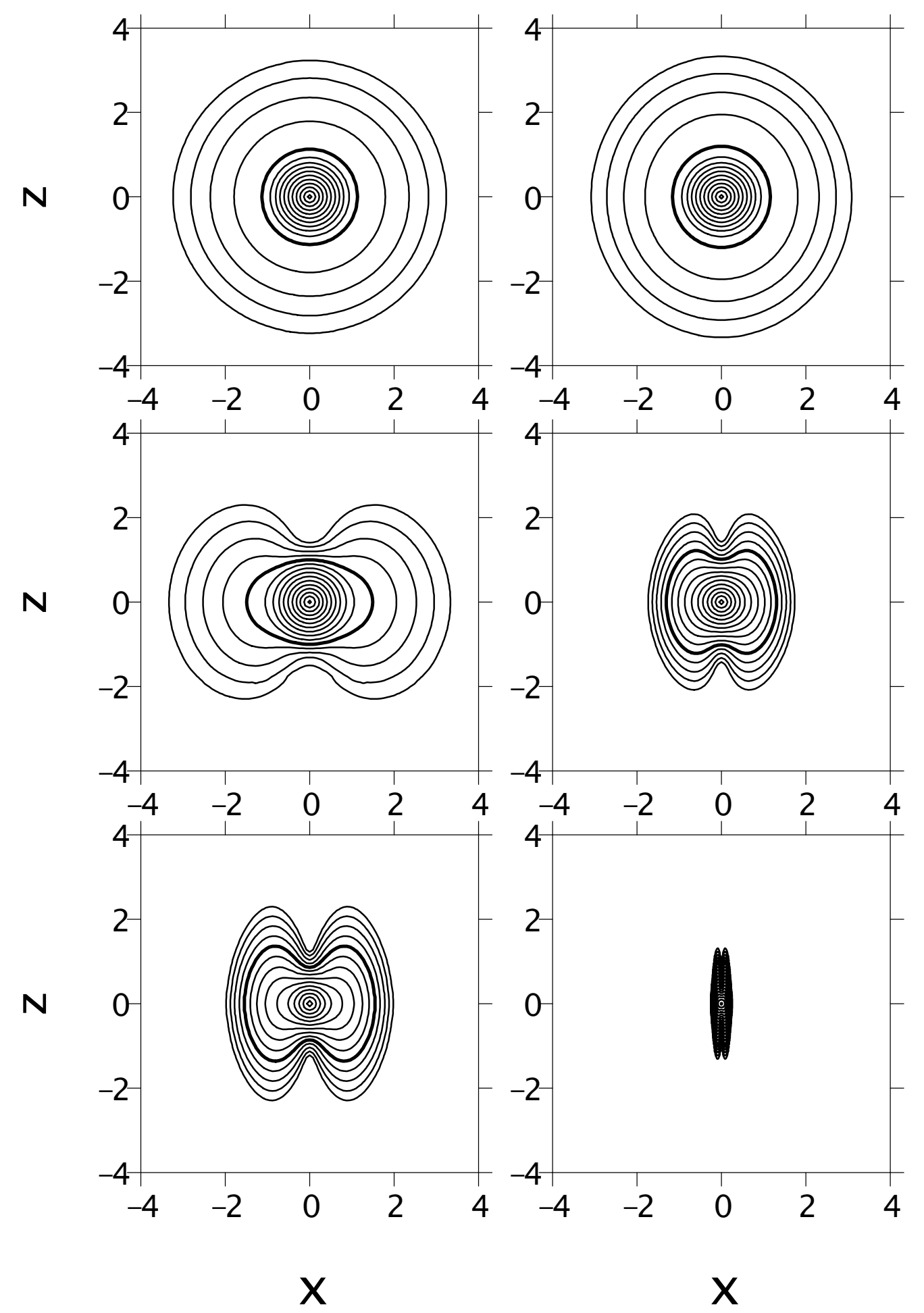

Figure 7 